\title{
Current status of presurgical infant orthopaedic treatment for cleft lip and palate patients: A critical review
}

\author{
P. Priyanka Niranjane, R. H. Kamble, S. Pallavi Diagavane, S. Sunita Shrivastav, Puneet Batra ${ }^{1}$, \\ S. D. Vasudevan, Pushkar Patil ${ }^{2}$ \\ Department of Orthodontics and Dentofacial Orthopedics, Sharad Pawar Dental College and Hospital, Sawangi, Wardha, \\ ${ }^{1}$ Department of Orthodontics and Dentofacial Orthopedics, Institute of Dental Studies and Technologies, Modinagar, Kadrabad, \\ Meerut, Uttar Pradesh, ${ }^{2}$ Private Practise, Dhule, Maharashtra, India
}

Address for correspondence: Dr Priyanka Niranjane, c/o Mr. Pravin Chahare, Chintamani Apartments, Sukhakarta Nagari, Sawangi (Meghe), Wardha, Maharashtra, India. E-mail: drpriyankaniranjane@yahoo.com

\section{ABSTRACT}

Rehabilitation of cleft lip and palate (CLP) patients is a challenge for all the concerned members of the cleft team, and various treatment modalities have been attempted to obtain aesthetic results. Presurgical infant orthopaedics (PSIO) was introduced to reshape alveolar and nasal segments prior to surgical repair of cleft lip. However, literature reports lot of controversy regarding the use of PSIO in patients with CLP. Evaluation of long-term results of PSIO can provide scientific evidence on the efficacy and usefulness of PSIO in CLP patients. The aim was to assess the scientific evidence on the efficiency of PSIO appliances in patients with CLP and to critically analyse the current status of PSIO. A PubMed search was performed using the terms PSIO, presurgical nasoalveolar moulding and its long-term results and related articles were selected for the review. The documented studies report no beneficial effect of PSIO on maxillary arch dimensions, facial aesthetics and in the subsequent development of dentition and occlusion in CLP patients. Nasal moulding seems to be more beneficial and effective in unilateral cleft lip and palate patients with better long-term results.

\section{KEY WORDS}

Bilateral cleft lip and palate; long-term results; nasoalveolar moulding; presurgical infant orthopaedics; unilateral cleft lip and palate

\section{INTRODUCTION}

acial clefting is the second most common congenital deformity. Over the years, various treatment modalities have been attempted in these patients so

\begin{tabular}{|l|l|}
\hline \multicolumn{2}{|c|}{ Access this article online } \\
\hline Quick Response Code: & Website: \\
\hline & www.ijps.org \\
\cline { 2 - 3 } & Dol: \\
\hline
\end{tabular}

as to achieve a satisfactory outcome. Infant orthopaedic (IO) treatment was first introduced by McNeil ${ }^{[1]}$ and was further improved by others. ${ }^{[2-6]}$ From McNeil's concept of alveolar moulding to concept of nasoalveolar moulding (NAM) many changes have also taken place in appliance design. In 1999, Grayson et al. ${ }^{[7]}$ described a new technique to presurgically mould the lip, alveolus and nose in infants born with cleft lip and palate (CLP). The concept of NAM works on Matsuo's principle; ${ }^{[8]}$ that the nasal cartilage could be moulded due to increased plasticity concurrent to increased levels of maternal oestrogen, if treatment is initiated within 6 weeks of life. Presurgical nasoalveolar moulding (PNAM) appliances have been in use as a new 
approach to traditional presurgical infant orthopaedics (TPSIO). ${ }^{[7,9-12]}$ The NAM appliance consists of an intraoral moulding plate with nasal stents to mould the alveolar ridge and nasal cartilage concurrently.

Some of the major advantages of TPSIO are claimed to be the improvement in arch form, facilitation of surgical closure, and thus improvement of aesthetic outcome, facilitation of feeding, and improvement of speech. ${ }^{[13-18]}$ Advocates of PNAM have stated that, beside other advantages of traditional plates, the main objectives of PNAM appliances are improving nasal symmetry and lip aesthetics while elongating the columella and correcting nasal cartilage deformity..$^{[7,10,19-22]}$ On the other hand, opponents have stated that all types of presurgical infant orthopaedics (PSIO) approaches are complex and expensive and might have an adverse effect on maxillary growth. None of these claims are evidence based. ${ }^{[23,24,16,17]}$ As there is not yet a definitive conclusion on the subject, a review of PSIO seems to be warranted.

Evaluation of long-term outcomes of different treatment protocols has become more valuable because it is well known that the definitive outcome of treatment in patients with CLP cannot be established until facial development is complete.

The aim of the present review is to assess the scientific evidence on the efficacy of PSIO appliances in patients with CLP and to critically analyse the current status of PSIO.

\section{METHODS}

A PubMed search was performed using the terms PSIO, PNAM and long-term effects and related articles were selected for the review. As the purpose of this review was to assess the long-term effects of PSIO in CLP patients, no efforts were made to study the effects of PSIO on unilateral cleft lip and palate (UCLP) and bilateral cleft lip and palate (BCLP) patients separately. A minimum 3-year follow-up was decided, and no restrictions were made regarding the type of PSIO appliance. Similarly as surgical times and techniques affect treatment outcomes, studies on PSIO using control groups treated with different surgical methods also were not included in the study.

Long-term effects of PSIO can be studied as under'Table 1]: - Effects of PSIO on maxillary growth
- Effects of PSIO on dentition and occlusion

- Effects of PSIO on facial appearance

- Effects of PSIO on nasal symmetry

- Effects of PSIO on speech.

\section{Long-term effects of presurgical infant orthopaedics on facial growth, maxillary arch, and dentition and occlusion}

Studies, published in the 80s and 90s, dealt mainly with the effect of PSIO on maxillary arch dimensions with little attention on other aspects of the maxillofacial growth. Most studies, however, were retrospective, had a small sample size, lacked a control group with UCLP without PSIO, had no clear outcome measures, or did not take confounding variables into account. ${ }^{[25-33]}$ Only a few studies could be found that had an adequate research design to investigate the effects of PSIO.

Mishima et al. ${ }^{[34-36]}$ using a two-group quasirandomised design with a control group $(n=8)$ children without PSIO, found that prior to lip surgery the maxillary segments in the PSIO group $(n=12)$ moved towards the midline, and in the non-PSIO group, the maxillary segments remained in the same position or displaced laterally. At 18 months of age, the curvature of palatal shelves was less steep, and there was less arch collapse in PSIO group when compared with control group. At 4-year of age, the distance between deciduous canines and second deciduous molars was larger in the PSIO group; other variables did not differ between the two groups.

A three-centre, randomised, prospective clinical trial in Netherlands. ${ }^{[37,38]}$ (Dutchcleft) evaluating the effects of PSIO showed comparable differences in maxillary arch dimensions between PSIO $(n=27)$ and the non-PSIO group $(n=27)$ until lip surgery at 15 weeks of age. However, at 12 months of age (prior to soft palate closure), no differences with respect to maxillary dimensions existed anymore between the two groups.

In the Dutchcleft studies, facial growth, maxillary arch dimension, and occlusion were further assessed at the age of 4 and 6-year. ${ }^{[39-41]}$ No significant differences were found in any of the variables between the $\left(\mathrm{IO}^{+}\right)$and $\left(\mathrm{IO}^{-}\right)$ groups when the occlusion was assessed by 5 -year-old index and Huddart score. Arch width, arch depth, arch length, arch form, and the vertical position of the lesser segment were measured. Authors found no clinically significant differences between $\left(\mathrm{IO}^{+}\right)$and $\left(\mathrm{IO}^{-}\right)$for any of the variables. 
When comparing facial growth and occlusion, the centres that practiced PSIO did not show demonstrable advantages in patients with UCLP treated with different types of PSIO appliances. ${ }^{[26,42-44]}$

Lee et al. ${ }^{[45]}$ assessed the effects of NAM and gingivoperiosteoplasty (GPP) in 20 UCLP patients at 6-year and at 11.5-year that is, at pre-pubertal age using lateral cephalogram and found that midface growth in sagittal or vertical planes (up to the age of 9-13 years) was not affected by pre-surgical alveolar moulding and GPP.

Adali et $a l .{ }^{[46]}$ studied the effect of PNAM on arch circumferenceand arch formintransverse, anteroposterior, and vertical dimensions on study model sets of 75 UCLP infants using Reflex Microscope and concluded that presurgical orthopaedics did not produce any significant effect on the arch form.

Ringdahl (2011) ${ }^{[47]}$ studied the long-term effects of NAM on mid face growth in 28 UCLP patients using photographs, study models and lateral cephalograms and concluded that there was no significant difference between moulding and nonmoulding groups in Goslon score.

Ross and MacNamera ${ }^{[13]}$ analysed the long-term effect of PSIO on facial aesthetics in 20 complete BCLP patients using facial photographs and concluded that PSIO has no lasting effect on lip, nose and facial aesthetics and does not alter the need for subsequent revisionary surgery.

The Dutchcleft studies ${ }^{[39-41]}$ also concluded that there was no clinically relevant effect of IO with passive plates on facial growth until the age of 6-year.

\section{Long-term effects of presurgical infant orthopedics on nasal symmetry and nasolabial appearance}

The correction of nasal deformity continues to be the greatest challenge in CLP cases. ${ }^{[4]}$ It is complex and affects the shape of the nose in all three planes of space. Matsuo et al ${ }^{[8]}$ and Matsuo and Hirose ${ }^{[48]}$ were the first to describe pre-surgical moulding of nasal cartilage in the neonate. Grayson et al. developed an appliance with a nasal extension attached to the anterior portion of an acrylic alveolar moulding plate, which marked the advent of PNAM appliances. Grayson et al. ${ }^{[49,50]}$ designed their nasal stent to extend from the anterior flange of an orthopaedic appliance used to mould the cleft alveolar segments. The resultant effect was NAM appliance and could be inserted as early as possible after birth. It is suggested that the main difference of NAM from traditional presurgical infant orthopaedics is the reshaping of nasal cartilage and providing aesthetic benefits in terms of nasal tip and alar symmetry. ${ }^{[7,-12]}$ Thus, nasal symmetry was investigated in most of the studies on NAM. ${ }^{[59]}$

In a 6-year follow-up by Bennun et al. ${ }^{[51]}$ to compare growth and cosmetic results of 97 UCLP patients using plaster models by using surface impressions of the babies revealed a better and permanent nostril symmetry, increase in the columellar length and no alar cartilage luxation in patients who had the nasal component.

Maull et al..$^{[9]}$ evaluated long-term effects of NAM on three-dimensional nasal shape in unilateral clefts and found a significant change in nasal symmetry that was also maintained long term in early childhood. However, a major limitation of this study was that the children were not fully grown, and the control group was not age matched.

Liou et al. ${ }^{[19]}$ assessed the progressive changes of nasal symmetry with growth after NAM in 25 UCLP patients at the initial visit (T1), after NAM (T2), 1 week (T3), 1-year (T4), 2-year (T5), and 3-year (T6) after cheiloplasty using standard basilar view photographs. Nasal symmetry was assessed by the "quantity of asymmetry" (in millimetres), that was the linear difference of each measurement between the cleft and non-cleft (cleft-non-cleft). The quantity of asymmetry revealed that nasal symmetry improved after NAM (T1-T2) and further corrected after cheiloplasty (T2-T3), but relapsed in $1^{\text {st }}$ year (T3-T4) and then remained stable in $2^{\text {nd }}$ and $3^{\text {rd }}$ years after surgery (T4-T6). Nasal symmetry was acceptable after 3-year post-operatively. The relapse in nasal asymmetry was the result of a significant differential growth/relapsed between the cleft and non-cleft sides in the $1^{\text {st }}$ year post-operatively. On the cleft side, the growth of nostril height and nasal dome height was significantly less, the columella length shortened (relapsed) significantly, and the growth of nostril width and nasal basal width was significantly less than on the non-cleft side. The relapse stopped, and the nasal growth was the same between the cleft and non-cleft sides in the second and $3^{\text {rd }}$ year. To compensate for relapse resulting from the differential growth in the $1^{\text {st }}$ year post-operatively, the authors have recommended narrowing down the alveolar cleft by NAM, overcorrecting the nasal vertical dimension 
surgically, and maintaining the surgical results by using a nasal conformer.

Lee et al. ${ }^{[20]}$ presented a series of 26 patients with bilateral CLP treated with a NAM protocol and stated that non-surgical columellar elongation with NAM followed by primary retrograde nasal reconstruction restored columellar length to normal by 3-year and significantly reduced the need for nasal reconstruction beyond their initial repair

A 9-year follow-up by Barillas et al. ${ }^{[52]}$ using stone cast measurements in 25 UCLP patients revealed that the nasal symmetry was improved by PNAM and was maintained at 9-year.

Sulaiman et al. ${ }^{[53]}$ did a 15 -year follow-up of pre-surgical orthopaedics, followed by primary correction for unilateral cleft lip nose in program SEHATI in Indonesia and found that the nostril height and width ratio and the height of the alar groove were significantly improved post-operatively and maintained for 15-year, but also stated that repositioning of nasal cartilage at infancy might not eliminate need for secondary correction after puberty.

A meta-analysis performed to study the effect of PNAM in unilateral cleft on nasal symmetry by van der Heijden et al. ${ }^{[54]}$ revealed that the results of NAM were inconsistent regarding changes in nasal symmetry; however, there was a trend towards a positive effect.

\section{Long term effects of presurgical infant orthopedics on speech}

Karling et al. ${ }^{[55]}$ evaluated the effect of T traction on speech in unilateral, bilateral cleft patients comparing them with the control group of non-cleft subjects and found no significant differences between the groups. However, the difference between mean ages of the groups (10.6year forliO $\left.{ }^{+}\right]$and 17.6-year forl' $\left.{ }^{-}\right]$) should be taken into consideration when evaluating this study.

Suzuki et al. ${ }^{[56]}$ studied the effect of pre-operative orthopaedic plate on articulatory function in 17 CLP children. Speech was assessed by speech therapists 3-year 11 months after palatoplasty. The results indicated that the continuous use of orthopaedic plate was effective in preventing palatalized articulation.

Konst et al. ${ }^{[57]}$ evaluated language skills of children between the ages of 2 and 6-year. The results showed that the early positive effects of PSIO changed over time, and no differences were found in language development between the two groups at the age of 6-year. However, the low follow-up rate reduced the level of evidence of this randomised controlled trial (RCT).

\section{DISCUSSION}

The effects of PSIO remains a subject of controversy in the medical literature. In the current review, the long term effects of PSIO treatment were examined, mainly with respect to the passive type of appliances. From the literature reports on the effects of PSIO on maxillary arch, dentition and occlusion, it seems that PSIO has no positive effect on maxillary arch dimensions and also does not improves the dentition and occlusion of UCLP children. The effects of PSIO on speech and language development also seems to be inconclusive although maxillofacial growth and speech are greatly influenced by the type, time and number of surgical procedures and also by the skill of performing surgeon. The only reported systematic review on effects of PSIO and its long-term advantages was released by Uzel and Alparslan. ${ }^{[58]}$ The authors concluded that until the age of 6 , there were no positive effects on factors like facial growth, maxillary arch dimension, or occlusion when treatment included passive IO appliances. However, the authors made the distinction between PSIO and NAM appliances, stating that their review yielded the conclusion that nasal symmetry was improved with NAM. A systematic review by van der Heijden et al. ${ }^{[54]}$ to quantify the long term effect of PNAM on nasal symmetry in unilateral cleft also reported a trend toward a positive effect of PNAM on nasal symmetry. However Chang et al. ${ }^{[59]}$ suggested that NAM alone could not provide nostril symmetry in the long-term and that overcorrection of $20 \%$ maintained nostril height after 5-year. Use of postsurgical nasal splint appliances for at least 6 months postoperatively have been advocated by Yeow et al. ${ }^{[60]}$ and Chang et al. ${ }^{[59]}$ to prevent relapse following NAM. These nasal splints help maintain the alar cartilage height and prevent collapse during scar healing and beyond. Nasal and facial anatomy and texture corresponding to ethnic descent, could be an important factor for remodeling a nose in the desired shape. Similarly skills of the dentist, orthodontist, and surgeon could also be a decisive factor in achieving improved nasal symmetry.

The Eurocleft study ${ }^{[61]}$ showed that centres with better outcomes were seen when there were few surgeons with 
Niranjane, et al.: Current status of presurgical infant orthopaedics

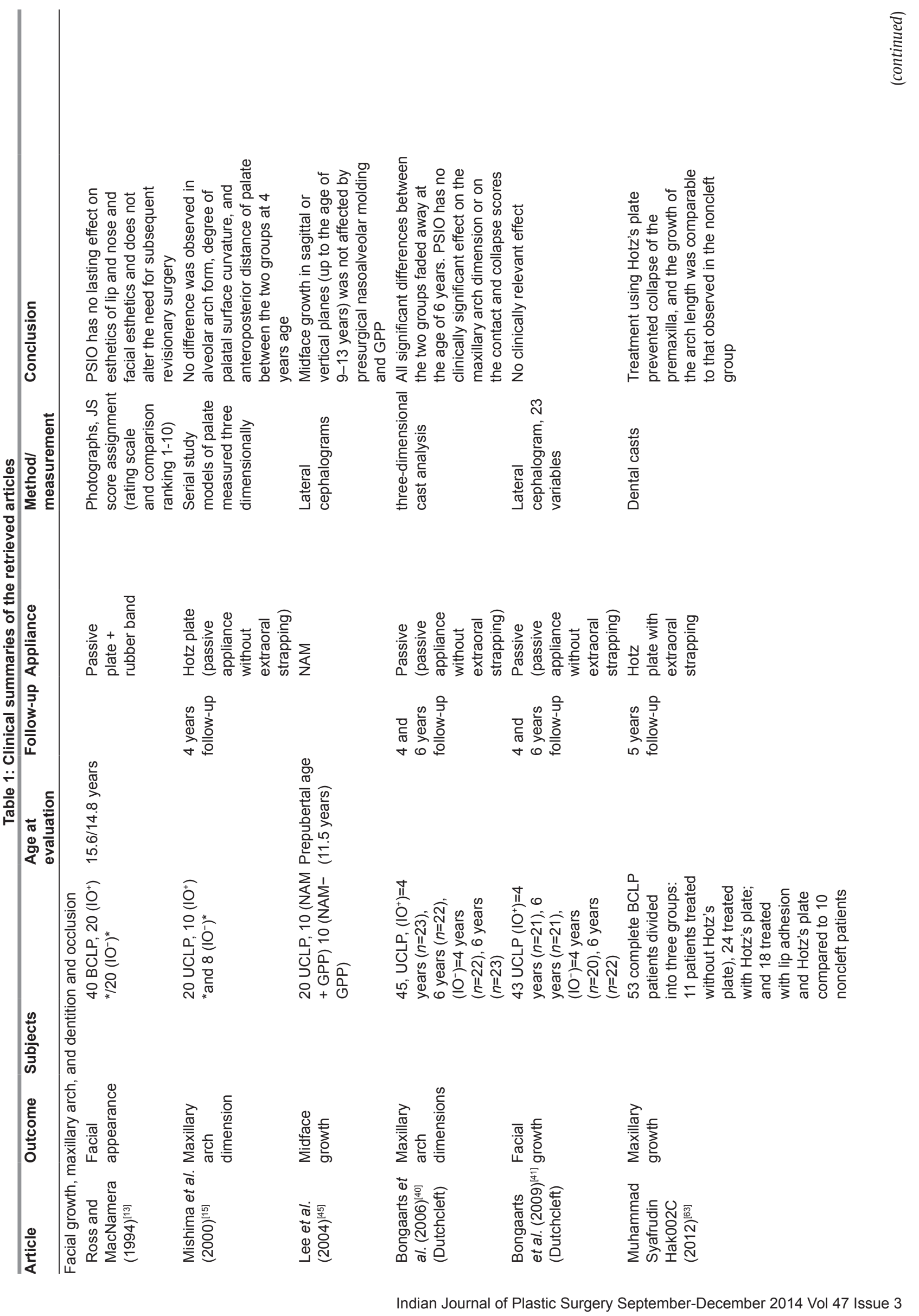




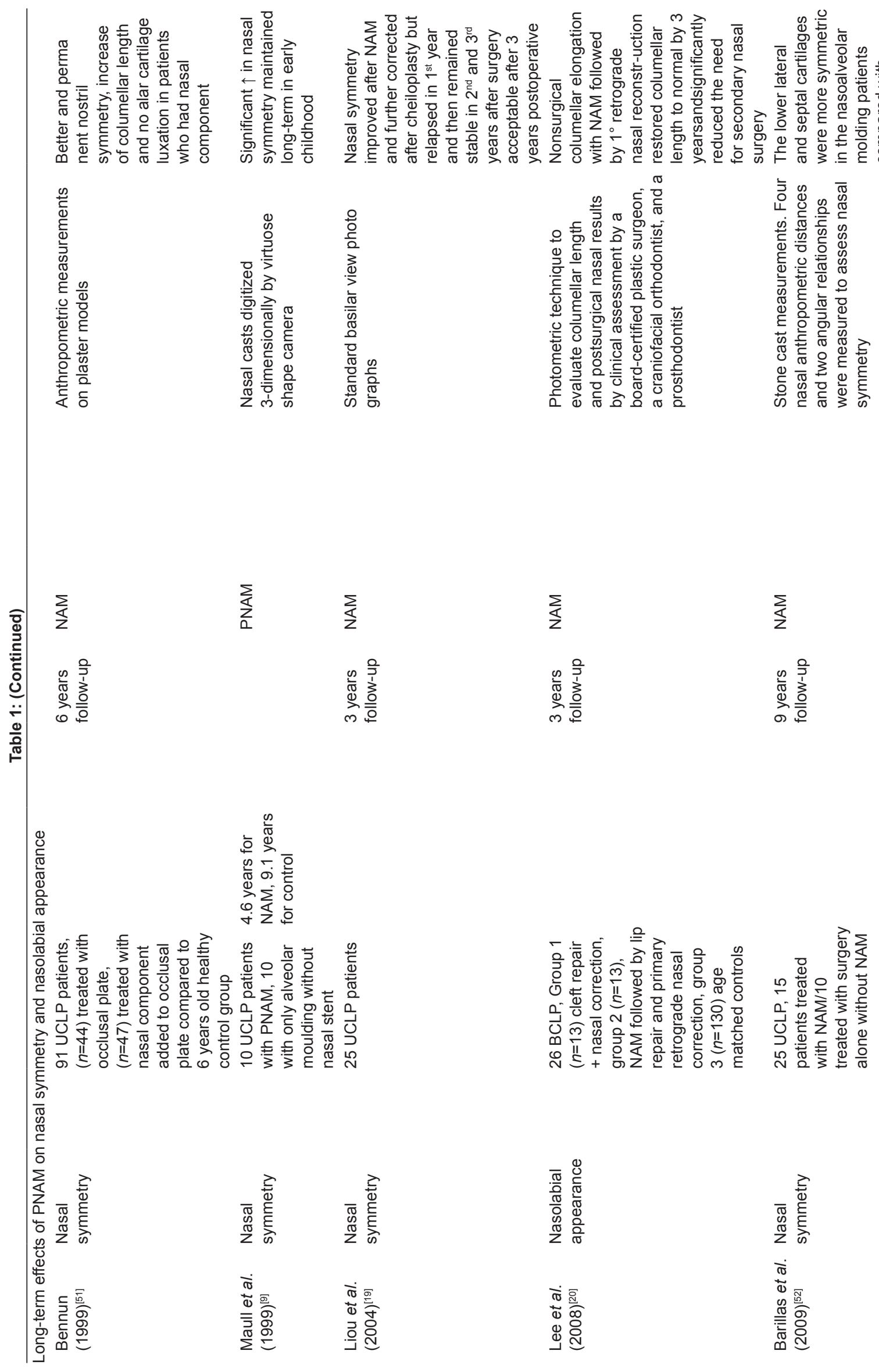


Niranjane, et al.: Current status of presurgical infant orthopaedics

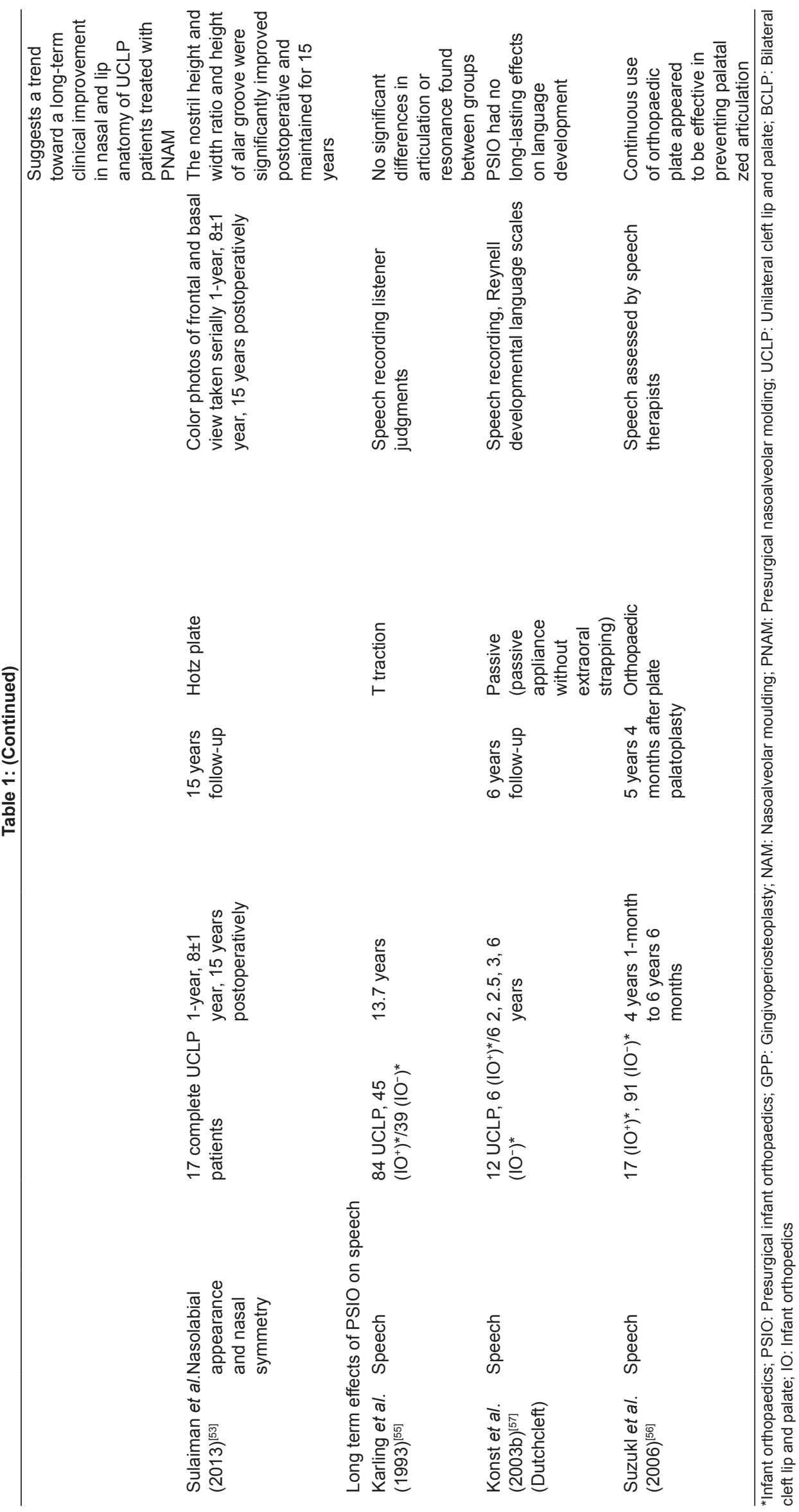


some differences in technique and timing, single layer cranially based vomer flap to close the anterior palate at the time of lip repair, no pre-surgical orthopedics, and delayed closure of hard palate at age 9. Conversely centres with lower ranking utilized active pre-surgical orthopedic treatment (extraoral strapping and nostril retraction, had many surgeons, utilized primary bone gragting and performed secondary revisions early. Similarly nearly identical conclusions were reached in the Americleft study ${ }^{[2]}$ regarding the association of more favorable outcomes with standard, simpler, and less burdensome protocols without PSIO, without primary bone grafting, and fewer surgeons.

Very few studies evaluating the long term effects of PSIO in BCLP patients can be found in the literature due to the low prevalence of BCLP. Bilateral cleft lip - cleft palate NAM is designed for nonsurgical columella elongation, orthopedic retraction of pre-maxilla and molding of the posterior lateral alveolar ridges to an appropriate width to accept the pre-maxilla. 3 long term studies evaluating the efficacy of PSIO in BCLP patients have been included in the review out of which Ross and MacNamera ${ }^{[13]}$ reported that conservative PSIO for BCLP patients does not have lasting effects on the esthetics of the lip and nose while Lee et al. ${ }^{[20]}$ stressed that nasoalveoalr molding combined with retrograde nasal reconstruction improves the quality of reconstructive outcome and decreases the number of surgical procedures. Hak et al. ${ }^{[63]}$ in a prospective longitudinal evaluation on 53 BCLP patients also showed that treatment with Hotz's plate until about 18 months of age prevented collapse of the pre-maxilla and supported the growth of dental arch length until the age of 5-year compared to non-cleft patients.

Thus unfortunately, at this time there is not a good body of evidence to support a statement either for or against using PSIO. However, a trend in the literature is being seen to support nasal moulding, but long-term studies are needed to analyse whether nasal moulding truly reduces the need for future nasal revision or other health care costs with age.

\section{Limitations of the review}

One of the greatest problems faced in PSIO research is small sample size, due to the relatively low prevalence of UCLP. Multicentre studies have aimed to overcome this by pooling different samples, thereby introducing additional problems with comparisons, especially regarding the heterogeneity of samples and variations in the number and experience of surgeons (Roberts-Harry et al. ${ }^{[64]}$ Prahl et al. ${ }^{[16]}$ The Euro cleft studies ${ }^{[44]}$ have the longest follow-up in the literature (17-year), but direct comparison between $\left(\mathrm{PSIO}^{+}\right)$and $\left(\mathrm{PSIO}^{-}\right)$could not be made in the intercentre studies due to the differences in the treatment protocols. Thus, the effects of PSIO in adults remain unclear. Moreover, each study used a unique combination of population and treatment duration, which makes comparison of every single aspect of treatment impossible. In addition, this meta-analysis has not taken into account the different techniques of PSIO, timings of surgery and surgical techniques, which would have made comparison of the results even more difficult. All these aspects together have made conclusions of the results of the studies by means of the intended meta-analysis impossible.

\section{CONCLUSION}

The current review article is an attempt to critically analyse the literature regarding the effects of PSIO and to comment on the current status of PSIO. The documented studies report no beneficial effects of PSIO on maxillary arch dimensions and in the subsequent development of dentition and occlusion in UCLP patients. The studies on the effect of PSIO on speech are also inconclusive. Based on the literature review, nasal moulding seems to be more beneficial and effective with better long term results, however the effects on nasal and alveolar moulding needs to be studied further to assess the long term beneficial effects. In the future, well-designed RCTs with long term follow-up should be undertaken in order to provide additional evidence to confirm or reject PSIO effectiveness.

\section{REFERENCES}

1. McNeil CK. Congenital oral deformities. Br Dent J 1956;18: 191-8.

2. Hotz M, Gnoinski W. Comprehensive care of cleft lip and palate children at Zürich university: A preliminary report. Am J Orthod 1976;70:481-504

3. Hotz MM, Gnoinski WM. Effects of early maxillary orthopaedics in coordination with delayed surgery for cleft lip and palate. J Maxillofac Surg 1979;7:201-10.

4. Huddart AG. Presurgical changes in unilateral cleft palate subjects. Cleft Palate J 1979;16:147-57.

5. Weil J. Orthopaedic growth guidance and stimulation for patients with cleft lip and palate. Scand J Plast Reconstr Surg Hand Surg 1987;21:57-63.

6. Hochban W, Austermann KH. Presurgical orthopaedic treatment using hard plates. J Craniomaxillofac Surg 1989;17 Suppl 1:2-4.

7. Grayson BH, Santiago PE, Brecht LE, Cutting CB. Presurgical nasoalveolar molding in infants with cleft lip and palate. Cleft Palate Craniofac J 1999;36:486-98. 
8. Matsuo K, Hirose T, Otagiri T, Norose N. Repair of cleft lip with nonsurgical correction of nasal deformity in the early neonatal period. Plast Reconstr Surg 1989;83:25-31.

9. Maull DJ, Grayson BH, Cutting CB, Brecht LL, Bookstein FL, Khorrambadi $\mathrm{D}$, et al. Long-term effects of nasoalveolar molding on three-dimensional nasal shape in unilateral clefts. Cleft Palate Craniofac J 1999;36:391-7.

10. Grayson $\mathrm{BH}$, Cutting $\mathrm{CB}$. Presurgical nasoalveolar orthopedic molding in primary correction of the nose, lip, and alveolus of infants born with unilateral and bilateral clefts. Cleft Palate Craniofac J 2001;38:193-8.

11. Suri S, Tompson BD. A modified muscle-activated maxillary orthopedic appliance for presurgical nasoalveolar molding in infants with unilateral cleft lip and palate. Cleft Palate Craniofac J 2004;41:225-9.

12. Singh GD, Levy-Bercowski D, Santiago PE. Three-dimensional nasal changes following nasoalveolar molding in patients with unilateral cleft lip and palate: Geometric morphometrics. Cleft Palate Craniofac J 2005;42:403-9.

13. Ross RB, MacNamera MC. Effect of presurgical infant orthopedics on facial esthetics in complete bilateral cleft lip and palate. Cleft Palate Craniofac J 1994;31:68-73.

14. Kuijpers-Jagtman AM, Ross EL Jr. The influence of surgery and orthopedic treatment on maxillofacial growth and maxillary arch development in patients treated for orofacial clefts. Cleft Palate Craniofac J 2000;37:527-39.

15. Mishima K, Mori Y, Sugahara T, Minami K, Sakuda M. Comparison between palatal configurations in UCLP infants with and without a Hotz plate until four years of age. Cleft Palate Craniofac J 2000;37:185-90.

16. Prahl C, Kuijpers-Jagtman AM, van't Hof MA, Prahl-Andersen B. A randomised prospective clinical trial into the effect of infant orthopaedics on maxillary arch dimensions in unilateral cleft lip and palate (Dutchcleft). Eur J Oral Sci 2001;109:297-305.

17. Konst EM, Prahl C, Weersink-Braks H, De Boo T, Prahl-Andersen B, Kuijpers-Jagtman AM, et al. Cost-effectiveness of infant orthopedic treatment regarding speech in patients with complete unilateral cleft lip and palate: A randomized three-center trial in the Netherlands (Dutchcleft). Cleft Palate Craniofac J 2004;41:71-7.

18. Berkowitz S. Cleft Lip and Palate. $2^{\text {nd }}$ ed. Berlin: Springer-Verlag; 2006.

19. Liou EJ, Subramanian M, Chen PK, Huang CS. The progressive changes of nasal symmetry and growth after nasoalveolar molding: A three-year follow-up study. Plast Reconstr Surg 2004; 114:858-64.

20. Lee CT, Garfinkle JS, Warren SM, Brecht LE, Cutting CB, Grayson $\mathrm{BH}$. Nasoalveolar molding improves appearance of children with bilateral cleft lip-cleft palate. Plast Reconstr Surg 2008;122:1131-7.

21. Ezzat CF, Chavarria C, Teichgraeber JF, Chen JW, Stratmann RG, Gateno J, et al. Presurgical nasoalveolar molding therapy for the treatment of unilateral cleft lip and palate: A preliminary study. Cleft Palate Craniofac J 2007;44:8-12.

22. Singh GD, Levy-Bercowski D, Yáñez MA, Santiago PE. Threedimensional facial morphology following surgical repair of unilateral cleft lip and palate in patients after nasoalveolar molding. Orthod Craniofac Res 2007;10:161-6.

23. Severens JL, Prahl C, Kuijpers-Jagtman AM, Prahl-Andersen B. Short-term cost-effectiveness analysis of presurgical orthopedic treatment in children with complete unilateral cleft lip and palate. Cleft Palate Craniofac J 1998;35:222-6.

24. Prahl-Andersen B. Dental treatment of predental and infant patients with clefts and craniofacial anomalies. Cleft Palate Craniofac J 2000;37:528-32.
25. HuddartAG. The effect of form and dimension on the management of the maxillary arch in unilateral cleft lip and palate conditions. Scand J Plast Reconstr Surg Hand Surg 1987;21:53-6.

26. Ross RB. Treatment variables affecting facial growth in unilateral cleft lip and palate. Part 2: Presurgical orthopedics. Cleft Palate Craniofac J 1987;24:24-32.

27. Gnoinski WM. Orofacial development up to age 15 in UCLP cases treated according to the current Zurich approach. In: Pfeiffer G, editor. Craniofacial Anomalies and Clefts of Lip, Alveolus and Palate. Principles of Treatment, Long Term Results. Stuttgart: G. Thieme; 1989.

28. Kramer GJ, Hoeksma JB, Prahl-Andersen B. Early palatal changes in complete and incomplete cleft lip and/or palate. Acta Anat (Basel) 1992;144:202-12.

29. Kramer GJ, Hoeksma JB, Prahl-Andersen B. Palatal changes after lip surgery in different types of cleft lip and palate. Cleft Palate Craniofac J 1994;31:376-84.

30. Larson M, Sällström KO, Larson O, McWilliam J, Ideberg M. Morphologic effect of preoperative maxillofacial orthopedics (T-traction) on the maxilla in unilateral cleft lip and palate patients. Cleft Palate Craniofac J 1993;30:29-34.

31. Ball JV, DiBiase DD, Sommerlad BC. Transverse maxillary arch changes with the use of preoperative orthopedics in unilateral cleft palate infants. Cleft Palate Craniofac J 1995;32:483-8.

32. Kozelj V. The basis for presurgical orthopedic treatment of infants with unilateral complete cleft lip and palate. Cleft Palate Craniofac J 2000;37:26-32.

33. Opitz C, Kratzsch H. Maxilla dimension in patients with unilateral and bilateral cleft lip and palate. Changes from birth until palate surgery at age three. J Orofac Orthop 1997;58:110-23.

34. Mishima K, Sugahara T, Mori Y, Sakuda M. Three-dimensional comparison between the palatal forms in infants with complete unilateral cleft lip, alveolus, and palate with and without Hotz plate. Cleft Palate Craniofac J 1996;33:77-83.

35. Mishima K, Sugahara T, Mori Y, Sakuda M. Three-dimensional comparison between the palatal forms in complete unilateral cleft lip and palate with and without Hotz plate from cheiloplasty to palatoplasty. Cleft Palate Craniofac J 1996;33:312-7.

36. Mishima K, Sugahara T, Mori Y, Sakuda M. Comparison of palatal forms in complete unilateral cleft lip and palate infants with and without a Hotz plate. In: Lee ST, Huang M, editors. Transactions of the $8^{\text {th }}$ International Congress on Cleft Palate and Related Craniofacial Anomalies. Singapore: Stamford Press; 1997. p. 394-7.

37. Kuijpers-Jagtman AM, Prahl C. A study into the effects of presurgical orthopaedic treatment in complete unilateral cleft lip and palate patients. A three centre prospective clinical trial in Nijmegen, Amsterdam and Rotterdam. Interim analysis. Nijmegen: University Press; 1996.

38. Prahl C, Kuijpers-Jagtman AM, Prahl-Andersen B. Effect of presurgical infant orthopedics on maxillary arch dimensions. In: Lee ST, Huang $M$, editors. Transactions $8^{\text {th }}$ International Congress on Cleft Palate and Related Craniofacial Anomalies. Singapore: Stamford Press; 1997. p. 1026.

39. Bongaarts CA, Kuijpers-Jagtman AM, van't Hof MA, PrahlAndersen $B$. The effect of infant orthopedics on the occlusion of the deciduous dentition in children with complete unilateral cleft lip and palate (Dutchcleft). Cleft Palate Craniofac J 2004;41: 633-41.

40. Bongaarts CA, van't Hof MA, Prahl-Andersen B, Dirks IV, KuijpersJagtman AM. Infant orthopedics has no effect on maxillary arch dimensions in the deciduous dentition of children with complete unilateral cleft lip and palate (Dutchcleft). Cleft Palate Craniofac J 2006;43:665-72. 
41. Bongaarts CA, Prahl-Andersen B, Bronkhorst EM, Prahl C, Ongkosuwito EM, Borstlap WA, et al. Infant orthopedics and facial growth in complete unilateral cleft lip and palate until six years of age (Dutchcleft). Cleft Palate Craniofac J 2009;46: 654-63.

42. Mars M, Asher-McDade C, Brattström V, Dahl E, McWilliam J, Mølsted $\mathrm{K}$, et al. A six-center international study of treatment outcome in patients with clefts of the lip and palate: Part 3. Dental arch relationships. Cleft Palate Craniofac J 1992;29:405-8.

43. Mølsted K, Asher-McDade C, Brattström V, Dahl E, Mars M, McWilliam J, et al. A six-center international study of treatment outcome in patients with clefts of the lip and palate: Part 2. Craniofacial form and soft tissue profile. Cleft Palate Craniofac J 1992;29:398-404.

44. Mølsted K, Brattström V, Prahl-Andersen B, Shaw WC, Semb G. The Eurocleft study: Intercenter study of treatment outcome in patients with complete cleft lip and palate. Part 3: Dental arch relationships. Cleft Palate Craniofac J 2005;42:78-82.

45. Lee CT, Grayson BH, Cutting CB, Brecht LE, Lin WY. Prepubertal midface growth in unilateral cleft lip and palate following alveolar molding and gingivoperiosteoplasty. Cleft Palate Craniofac J 2004;41:375-80.

46. Adali N, Mars M, Petrie A, Noar J, Sommerlad B. Presurgical orthopedics has no effect on archform in unilateral cleft lip and palate. Cleft Palate Craniofac J 2012;49:5-13.

47. Ringdahl L. The Long-term effect of nasoalveolar molding on midface Growth and nasolabial esthetics in complete unilateral cleft lip and palate patients. Dissertation submitted to the South western University. Available from: http://www.gateway.proquest.com/ openurl\%3furl_ver=Z39.882004\%26res_dat=xri:pqdiss\%26rft_ val_fmt=info:ofi/fmt:kev:mtx:dissertation $\% 26 \mathrm{rft}$ dat=xri:pqdiss:1505904Last accessed September 2013].

48. Matsuo K, Hirose T. Preoperative non-surgical over-correction of cleft lip nasal deformity. Br J Plast Surg 1991;44:5-11.

49. Grayson BH, Cutting C, Wood R. Preoperative columella lengthening in bilateral cleft lip and palate. Plast Reconstr Surg 1993;92:1422-3.

50. Grayson BH, Santiago PE. Presurgical orthopaedics for cleft lip and palate. In: Aston SJ, Beasley RW, Thorne CHM, editors. Grabb and Smith's Plastic Surgery. $5^{\text {th }}$ ed. Philadelphia: Lippincott-Raven; 1997. p. 237-44.

51. Bennun RD, Perandones C, Sepliarsky VA, Chantiri SN, Aguirre MI, Dogliotti PL. Nonsurgical correction of nasal deformity in unilateral complete cleft lip: A 6-year follow-up. Plast Reconstr Surg 1999;104:616-30.

52. Barillas I, Dec W, Warren SM, Cutting CB, Grayson BH. Nasoalveolar molding improves long-term nasal symmetry in complete unilateral cleft lip-cleft palate patients. Plast Reconstr Surg 2009;123:1002-6.

53. Sulaiman FK, Haryanto IG, Hak S, Nakamura N, Sasaguri M, Ohishi M. Fifteen-Year Follow-Up Results of Presurgical Orthopedics Followed by Primary Correction for Unilateral Cleft
Lip Nose in Program SEHATI in Indonesia. Cleft Palate Craniofac J 2013;50:129-37.

54. van der Heijden P, Dijkstra PU, Stellingsma C, van der Laan BF, Korsten-Meijer AG, Goorhuis-Brouwer SM. Limited evidence for the effect of presurgical nasoalveolar molding in unilateral cleft on nasal symmetry: A call for unified research. Plast Reconstr Surg 2013;131:62e-71.

55. Karling J, Larson O, Leanderson R, Henningsson G. Speech in unilateral and bilateral cleft palate patients from Stockholm. Cleft Palate Craniofac J 1993;30:73-7.

56. Suzuki K, Yamazaki Y, Sezaki K, Nakakita N. The effect of preoperative use of an orthopedic plate on articulatory function in children with cleft lip and palate. Cleft Palate Craniofac J 2006;43:406-14.

57. Konst EM, Rietveld T, Peters HF, Kuijpers-Jagtman AM. Language skills of young children with unilateral cleft lip and palate following infant orthopedics: A randomized clinical trial. Cleft Palate Craniofac J 2003;40:356-62.

58. Uzel A, Alparslan ZN. Long-term effects of presurgical infant orthopedics in patients with cleft lip and palate: A systematic review. Cleft Palate Craniofac J 2011;48:587-95.

59. Chang CS, Por YC, Liou EJ, Chang CJ, Chen PK, Noordhoff MS. Long-term comparison of four techniques for obtaining nasal symmetry in unilateral complete cleft lip patients: A single surgeon's experience. Plast Reconstr Surg 2010;126:1276-84.

60. Yeow VK, Chen PK, Chen YR, Noordhoff SM. The use of nasal splints in the primary management of unilateral cleft nasal deformity. Plast Reconstr Surg 1999;103:1347-54.

61. Shaw WC, Brattström V, Mølsted K, Prahl-Andersen B, Roberts CT, Semb G. The Eurocleft study: Intercenter study of treatment outcome in patients with complete cleft lip and palate. Part 5: discussion and conclusions. Cleft Palate Craniofac J 2005;42: 93-8.

62. Russell K, Long RE Jr, Hathaway R, Daskalogiannakis J, Mercado A, Cohen M, et al. The Americleft study: An inter-center study of treatment outcomes for patients with unilateral cleft lip and palate part 5. General discussion and conclusions. Cleft Palate Craniofac J 2011;48:265-70.

63. Hak MS, Sasaguri M, Sulaiman FK, Hardono ET, Suzuki A, Nakamura S, et al. Longitudinal study of effect of hotz's plate and lip adhesion on maxillary growth in bilateral cleft lip and palate patients. Cleft Palate Craniofac J 2012;49:230-6.

64. Roberts-Harry D, Semb G, Hathorn I, Killingback N. Facial growth in patients with unilateral clefts of the lip and palate: $A$ two-center study. Cleft Palate Craniofac J 1996;33:489-93.

How to cite this article: Niranjane PP, Kamble RH, Diagavane SP, Shrivastav SS, Batra P, Vasudevan SD et al. Current status of presurgical infant orthopaedic treatment for cleft lip and palate patients: A critical review. Indian J Plast Surg 2014;47:293-302.

Source of Support: Nil, Conflict of Interest: None declared.

\section{Android Devices}
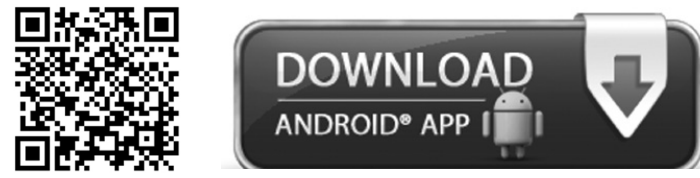

Use the QR code or download for android devices from:

http://www.mediafire.com/download/t7ugd37jug18ax0/IJPS.apk

Note: The android app is in beta testing and not available via google play store.

Please check the option 'Allow apps from unknown sources' to install IJPS app. 